\title{
Spin-dynamics simulation study of the field-induced phase transition for the spiral antiferromagnet $\mathrm{Ba}_{2} \mathrm{CuGe}_{2} \mathrm{O}_{7}$
}

\author{
Ran An and Xiaoping Wang \\ Department of Mathematics, The Hong Kong University of Science and Technology, Clear Water Bay, Hong Kong, China \\ Lei Zhou* \\ Surface Physics Laboratory (State Key Laboratory) and Physics Department, Fudan University, Shanghai 200433, China
}

(Received 19 July 2005; revised manuscript received 14 October 2005; published 12 December 2005)

\begin{abstract}
We perform spin-dynamics (SD) simulations to study the field induced incommensurate-to-commensurate phase transition for the spiral antiferromagnet $\mathrm{Ba}_{2} \mathrm{CuGe}_{2} \mathrm{O}_{7}$. Under an increasingly applied magnetic field, we find that the system undergoes a transition from a two-dimensional (2D) spiral state to a spin-flop state, passing through an intermediate phase. The simulations identify two order parameters to characterize this phase transition, of which one is the spiral period known previously, another is the root-mean-square value of a spin component. For the 2D spiral phase identified previously, our SD simulations yield essentially the same spin structures as previous continuum-field model studies when the applied field is very small, but quantitative differences exist when the field is not small. For the intermediate phase which was not fully understood by previous studies, our simulations reveal that its spin structure is a soliton-like state consisting of two spin-flop states connected by a three-dimensional spiral state.
\end{abstract}

DOI: 10.1103/PhysRevB.72.214422

PACS number(s): 75.25.+z, 75.40.Mg, 75.10.Hk, 75.50.Ee

\section{INTRODUCTION}

Due to the extremely weak interlayer coupling, $\mathrm{Ba}_{2} \mathrm{CuGe}_{2} \mathrm{O}_{7}$ is essentially a two-dimensional (2D) spin system with an antiferromagnetic nearest-neighbor coupling and a Dzyaloshinskii-Moriya (DM) anisotropy. ${ }^{1-3}$ In the absence of an external magnetic field, the ground state of $\mathrm{Ba}_{2} \mathrm{CuGe}_{2} \mathrm{O}_{7}$ is a $2 \mathrm{D}$ spin spiral (i.e., $S_{i}$ confined in a plane) with a well-defined spin-wave spectrum, resulted from a competition between the exchange coupling and the DM anisotropy. When an external magnetic field was applied perpendicular to the plane, Zheludev et al. observed experimentally that the ground state remained as an incommensurate spiral-like one when $H<H_{c}^{(1)}$, but became a commensurate spin-flop one when $H>H_{c}^{(2)}{ }^{4}$ Located in between the spiral and the spin-flop phase (i.e., $H_{c}^{(1)}<H<H_{c}^{(2)}$ ) is an intermediate phase whose nature is not clear from the experiments. ${ }^{4-8}$ These intriguing properties have attracted much attention theoretically. ${ }^{9}$ Based on a continuum-field model with parameter values extracted from experiments, the same group performed theoretical calculations to confirm that the ground state is indeed a spin spiral at a low field, and computed the spin-wave spectrum at $H=0 .{ }^{8}$ They also proposed that the intermediate phase might be a soliton lattice. ${ }^{6}$ Recently, Chovan et al. studied the same problem with a more delicate field theory based on a nonlinear $\sigma$ model, with which they could obtain the spin-wave spectra with an arbitrary $H$ in both the spiral and the spin-flop phases. ${ }^{10}$ The spin-wave analysis by Chovan et al. also reinforced the existence of an intermediate phase when $H_{c}^{(1)}<H<H_{c}^{(2)}$, since neither the spiral nor the spin-flop state was stable in that region. ${ }^{10}$ However, the exact configuration of this intermediate phase could not be deduced from the spin-wave analysis. ${ }^{10}$ The authors only suggested that a nonflat spiral was energetically more favorable when $H_{c}^{(1)}<H<H_{c}^{(2)}$, and a vortex state had a lower energy than a spin-flop state as $H$ $<H_{c}^{(2)} \cdot{ }^{10}$ It is thus our understanding that the nature of this intermediate phase is not yet completely clear.

The aim of the present paper is to study exactly how the spin configuration develops from a spin-spiral state to a spinflop state as the applied field increases. In particular, we want to reveal the spin configurations in the intermediate phase. In contrast to previous theories, which were all based on minimizing the energy functions of some continuum-field models, here we apply the spin-dynamics (SD) simulations ${ }^{11}$ on the realistic discrete spin lattices. For the present system, the spiral period at $H=0$ is about 36 times longer than the lattice constant (denoted by $a$ in the following), so that the application of a continuum-field model is well justified in the low field region. However, as $H$ increases, the spin direction may vary drastically in some situations so that the validity of a continuum-field model should be carefully reexamined. In contrast, the SD simulation is a highly reliable approach to search for the ground state of a complex spin system. Thus, in addition to providing information of the intermediate phase, our brute-force simulation results can also serve as some sort of justification of previous calculations based on continuum-field models.

The present paper is organized as follows. In Sec. II, we describe the adopted Hamiltonian for the system $\mathrm{Ba}_{2} \mathrm{CuGe}_{2} \mathrm{O}_{7}$, and the SD simulation approach. Section III is devoted to the discussion of the results, which include a complete description of how the spin configuration develops as the applied field increases, illustrations of typical spin configurations in three phase regions, and detailed comparisons with previous theories and experiments. We then summarize our results in Sec. IV. 


\section{HAMILTONIAN AND THE SPIN-DYNAMICS SIMULATION}

Following Ref. 8, the Hamiltonian of $\mathrm{Ba}_{2} \mathrm{CuGe}_{2} \mathrm{O}_{7}$ is taken as

$$
\mathcal{H}=\mathcal{H}^{(\mathrm{E})}+\mathcal{H}^{(\mathrm{DM})}+\mathcal{H}^{(\mathrm{KSEA})}+\mathcal{H}^{(\mathrm{Z})}
$$

where

$$
\mathcal{H}^{(\mathrm{E})}=\sum_{n, m} J\left(\vec{S}_{n, m} \cdot \vec{S}_{n+1, m}+\vec{S}_{n, m} \cdot \vec{S}_{n, m+1}\right)
$$

represents the Heisenberg antiferromagnetic exchange interaction with $J=0.96 \mathrm{meV}$ and the summation runs over all sites [denoted by $(n, m)]$ in the 2D lattice. The second term denotes the DM anisotropy ${ }^{1-3}$

$$
\mathcal{H}^{(\mathrm{DM})}=\sum_{n, m} D\left[\left(\vec{S}_{n, m} \times \vec{S}_{n+1, m}\right)_{y}+\left(\vec{S}_{n, m} \times \vec{S}_{n, m+1}\right)_{x}\right],
$$

where $D=0.1774 J$, taken from Ref. 10. The third term,

$$
\mathcal{H}^{(\mathrm{KSEA})}=\sum_{n, m} \frac{D^{2}}{2 J}\left(S_{n, m}^{y} S_{n+1, m}^{y}+S_{n, m}^{x} S_{n, m+1}^{x}\right)
$$

is the KSEA anisotropy term. ${ }^{8}$ The last term

$$
\mathcal{H}^{(\mathrm{z})}=-g \mu_{B} H \sum_{n, m} S_{n, m}^{z}
$$

describes the Zeeman interaction with an external field along the $z$ axis, where $H$ is the strength of the magnetic field, $\mu_{B}$ is the Bohr magneton and $g=2.474$ is the gyromagnetic ratio. ${ }^{7,10}$

We apply the classical SD simulation to search for the ground state of the system. Within this approach, the evolution of the classical spin vectors is described by the LandauLifshitz equation ${ }^{12,13}$

$$
\frac{d S_{n, m}}{d t}=-\vec{S}_{n, m} \times \vec{F}_{n, m}-\gamma \vec{S}_{n, m} \times\left(\vec{S}_{n, m} \times \vec{F}_{n, m}\right),
$$

where $\vec{F}_{n, m}$ is the local force experienced by a spin at lattice $(n, m)$, which can be derived from the Hamiltonian by

$$
\begin{aligned}
\vec{F}_{n, m}= & -\frac{\delta \mathcal{H}}{\delta \vec{S}_{n, m}}=-J\left(\begin{array}{l}
S_{n+1, m}^{x}+S_{n-1, m}^{x}+S_{n, m+1}^{x}+S_{n, m-1}^{x} \\
S_{n+1, m}^{y}+S_{n-1, m}^{y}+S_{n, m+1}^{y}+S_{n, m-1}^{y} \\
S_{n+1, m}^{z}+S_{n-1, m}^{z}+S_{n, m+1}^{z}+S_{n, m-1}^{z}
\end{array}\right) \\
& -D\left(\begin{array}{c}
S_{n-1, m}^{z}-S_{n+1, m}^{z} \\
S_{n, m+1}^{z}-S_{n, m-1}^{z} \\
S_{n+1, m}^{x}-S_{n-1, m}^{x}+S_{n, m-1}^{y}-S_{n, m+1}^{y}
\end{array}\right) \\
& -\frac{D^{2}}{2 J}\left(\begin{array}{c}
S_{n, m+1}^{x}+S_{n, m-1}^{x} \\
S_{n+1, m}^{y}+S_{n-1, m}^{y} \\
0
\end{array}\right)+H\left(\begin{array}{c}
0 \\
0 \\
1
\end{array}\right) .
\end{aligned}
$$

The second term in (6) is a damping term with $\gamma$ as a parameter. This term helps the system relax quickly to a steady state, but does not affect the structure of the obtained state. We took $\gamma=0.5$ in all our calculations. For a fixed external field $H$, we assume a random initial spin distribution and

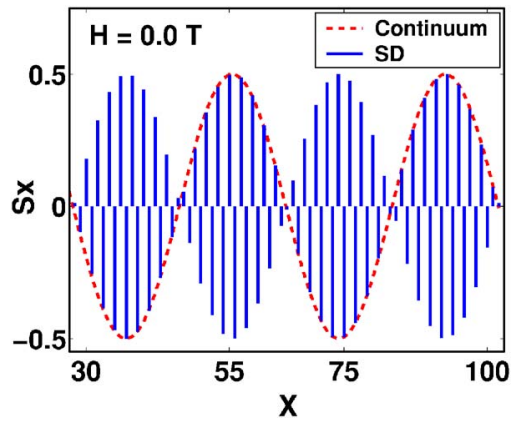

FIG. 1. (Color online) Part of spin configuration obtained by SD simulations (bars) at $H=0$, compared with the analytical results based on the continuum-field model (dashed line). Here the SD simulations were performed on a $370 \times 8$ lattice.

then iteratively solve Eq. (6) by the fourth order RungeKutta and Gauss-Seidel projection method. ${ }^{14,15}$ To minimize the risk of falling into a local minimum state, we used, for each case, several different random initial configurations and selected the true ground state by comparing the energies of the obtained states. The SD simulations have to be performed on a finite system. We note that the problem is essentially one dimensional in most situations. In order to save computational power, we modeled the system by $2 M$ chains (placed along the $x$ direction) each containing $2 N$ sites $(N \gg M)$. This approximation will be justified later by checking the convergence of the obtained results against varying $N$ and $M$. We adopted periodic boundary conditions for the $y$ direction$S_{n, 1}=S_{n, 2 M+1}, n=1,2, \ldots, 2 N$ - and the free boundary condition for the $x$ direction- $S_{0, m}=S_{2 N+1, m}=0, m=1,2, \ldots, 2 M$. By doing so, we actually only allow the spin spiral to propagate along the $x$ direction. We thus save huge computational resources and make the simulations more tractable.

As a benchmark test, we compare our simulation result with the available analytical solution at $H=0$. In the analytical approach, we assume the spin configuration as an ideal spiral

$$
\begin{gathered}
S_{n, m}^{x}=(-1)^{n+m} \sin (q n a+\phi), \quad S_{n, m}^{y}=0, \\
S_{n, m}^{z}=(-1)^{n+m} \cos (q n a+\phi),
\end{gathered}
$$

with $q$ as a parameter to be determined. Putting the above Ansatz to the energy function Eq. (1), we can determine the parameter $q$ by minimizing the energy function, $\partial \mathcal{H} / \partial q=0$, which leads to the following equation:

$$
q=a^{-1} \tan ^{-1}\left(\frac{D}{J}\right) .
$$

The period of the spiral can then be calculated by

$$
\lambda=\frac{2 \pi}{q}=\frac{2 \pi a}{\tan ^{-1}(D / J)} .
$$

The dashed line in Fig. 1 shows the envelope of the analytical solution Eq. (8). We then perform SD simulations to search for the ground state configuration at $H=0$. We have used several different initial spin configurations and found that the obtained final states were essentially the same. The 


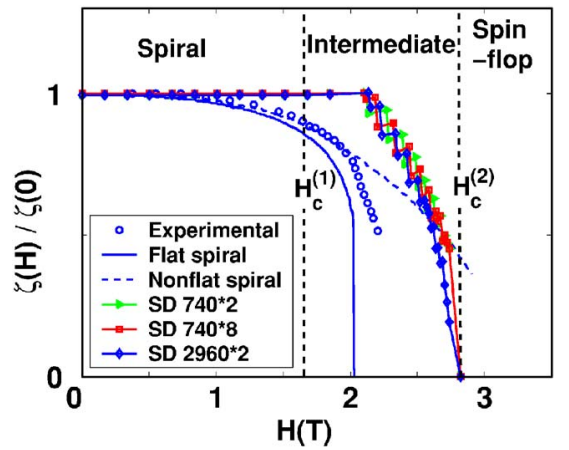

FIG. 2. (Color online) The incommensurability parameter $\zeta$, which is the inverse of the translation-invariant period, as a function of the external magnetic field $H$, obtained by SD simulations with various lattice sizes, by continuum-field model calculations, and by experimental measurements.

obtained state is a 2D spin spiral traveling along the $x$ direction, with $\vec{S}$ confined completely in the $x z$ plane (i.e., $S^{y} \equiv 0$ ). The spins in adjacent chains are perfectly antiparallel, and the period of the spiral is $\sim 36 a$, which is consistent with experiments and previous theories. To compare with the analytical solution, we depict the $x$ component of the spin vector $\left(S^{x}\right)$ as a function of the lattice site for one particular chain in Fig. 1 as solid bars. Obviously, the SD result is in excellent agreement with the analytical solution.

\section{RESULTS AND DISCUSSIONS}

Encouraged by the excellent agreement obtained at the zero $H$ case, we continue to study the ground states at nonzero $H$ fields. Both experiments and previous theories indicated that the ground state remained like a spiral with a period enlarged by the magnetic field. ${ }^{4-8}$ However, the role of a magnetic field is far more intriguing than just changing the period. Let us first assume that the ground state at a nonzero $H$ is still an ideal spin spiral as described by Eq. (8), but with a different period [determined by $q$ via Eq. (10)], then the Zeeman term becomes

$$
\mathcal{H}^{(\mathrm{z})}=-g \mu_{B} H \sum_{n, m} S_{n, m}^{z}=-g \mu_{B} H \sum_{n, m}(-1)^{m+n} \cos (q n a+\phi) .
$$

In an infinitely large system, the above term is exactly zero independent of the period, implying that the information of $H$ does not enter the treated problem at all. This suggests that the ground state at a nonzero $H$ field is not an ideal spin spiral.

The SD simulations can take account of the Zeeman interaction in an automatic manner, without any assumptions other than the finite sample size. After some efforts, we have successfully obtained the ground states at arbitrary $H$ cases through the SD simulations. In accordance with previous studies, we take $\zeta$, the inverse of the spiral period, as an order parameter to describe this incommensurate-tocommensurate phase transition. ${ }^{4-8,10,16}$ Figure 2 depicts $\zeta$ as a function of $H$ obtained through our SD simulations per-

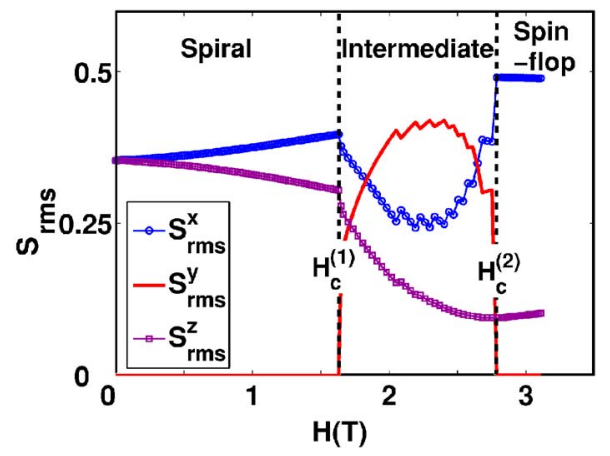

FIG. 3. (Color online) The root-mean-square values of three spin components $\left(S_{\mathrm{rms}}^{x}, S_{\mathrm{rms}}^{y}, S_{\mathrm{rms}}^{z}\right)$ as the functions of $H$.

formed on systems with different $N$ and $M$. The good agreements among different simulation results indicate that the finite-size effects are well controlled. We then compare our results with experimental ${ }^{4-8}$ and previous theoretical results, which were obtained by assuming that the ground state is a flat spiral ${ }^{7}$ or a nonflat spiral. ${ }^{10}$ Our results show that the ground state is indeed a spiral-like state as $H<H_{c}^{(1)}$ $=1.65 \mathrm{~T}$, is a spin-flop state as $H>H_{c}^{(2)}=2.79 \mathrm{~T}$, and the order parameter $\zeta$ decreases as $H$ increases. We also find an intermediate phase (i.e., $H_{c}^{(1)}<H<H_{c}^{(2)}$ ) in which the spin configuration is neither a spiral nor a spin-flop state. The lower critical field $H_{c}^{(1)}=1.65 \mathrm{~T}$ is in excellent agreement with both the experiment ${ }^{8}(\sim 1.7 \mathrm{~T})$ and the spin-wave analysis $^{10}(1.7 \mathrm{~T})$. The upper one, $H_{c}^{(2)}=2.79 \mathrm{~T}$, lies in between the values suggested by the experiment ${ }^{8}(\sim 2.2 \mathrm{~T})$ and the spin-wave analysis ${ }^{10}(\sim 2.9 \mathrm{~T})$. All these features are well consistent with experimental observations. ${ }^{4-8}$ However, we note that the quantitative agreement with the experimental data is not very good, especially in the high magnetic field regime. At present, we have not yet sorted out the exact reason accounting for such discrepancy. We suspect that the thermal fluctuations in the experiments and the ambiguities in determining the value of $J$ in the adopted Hamiltonian (1) are possibly responsible for this inconsistency.

The SD simulations show that the intermediate phase can be identified through a new order parameter $S_{\text {rms }}^{y}=\sqrt{\overline{\left(S^{y}\right)^{2}}}$, the root-mean-square (rms) value of the spin component along the $y$ direction. From the SD simulated spin configurations, we calculated $S_{\mathrm{rms}}^{x} S_{\mathrm{rms}}^{y}$ and $S_{\mathrm{rms}}^{z}$ as the functions of $H$ and show the results in Fig. 3. While the spin vectors are completely confined in the $x z$ plane (i.e., $S^{y} \equiv 0$ for all sites) in both the spiral and the spin-flop states, clearly the intermediate phase is characterized by the appearance of $S^{y}$.

We now illustrate the spin configurations in each phase region and show how the spin configuration evolves as $H$ increases. The solid bars in Fig. 4(a) denote the projected spin configuration for $H=1.55 \mathrm{~T}$. Compared with an ideal spiral with the same period [dashed line in Fig. 4(a)], we find the obtained spin configuration is no longer an ideal spiral, but rather a distorted one with more spin components lying along the $x$ axis (see also Fig. 3). We also calculated the spin configuration employing the continuum-field model ${ }^{10}$ under exactly the same $H$ field and show the result in the same 

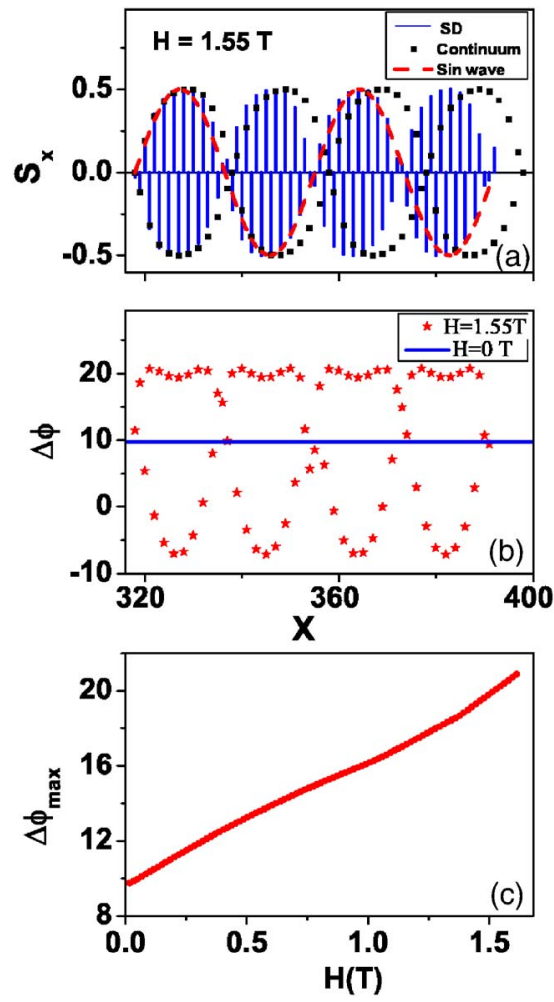

FIG. 4. (Color online) (a) Part of the spin configuration obtained by SD simulations (bars) in the case of $H=1.55 \mathrm{~T}$, compared with those obtained by continuum-field model calculations (solid symbols) and the ideal spiral solution (dashed line). (b) Angle difference between adjacent staggered spin vectors, $\Delta \phi$, as a function of the lattice site at $H=1.55 \mathrm{~T}$ (symbols) and $H=0$ (line). (c) The maximum angle difference $\left(\Delta \phi_{\max }\right)$ as a function of the $H$ field. Here the SD simulations were performed on a $370 \times 8$ lattice.

figure by solid symbols. The comparison between the SD result and the model one shows that surprisingly quantitative differences exist between the two configurations, and even the periods are different in the two cases. To explore the underlying physics, we computed the angle differences between two adjacent staggered spin vectors, $\Delta \phi_{n}=\phi_{n+1}-\phi_{n}$ $+\pi$, and depict $\Delta \phi_{n}$ as a function of lattice site $n$ in Fig. 4(b). As a reference, we also show the $\Delta \phi_{n} \sim n$ relation for the case of $H=0$ in the same figure as a solid line. Here, we have omitted the index $m$ for the sake of simplicity. It is interesting to note that $\Delta \phi_{n}$ has an odd-even oscillation when $H$ $\neq 0$, in consistency with previous continuum-field model analysis. ${ }^{7,10}$ More interestingly, we notice that, whereas, in the case of $H=0, \Delta \phi_{n}$ is uniform and is a small quantity $\left(\sim 10^{\circ}\right)$ everywhere; in the case $H=1.55 \mathrm{~T}, \Delta \phi_{n}$ could be quite large $\left(>20^{\circ}\right)$ at some lattice sites. This tendency is more significant as $H$ increases, as shown in Fig. 4(c), where the maximum angle difference $\Delta \phi_{n}$ is depicted as a function of $H$. Since the continuum-field approximation is justified only when the spin direction has a slow spatial variation, we conclude here that the continuum-field model becomes less accurate as $H$ increases, particularly when approaching the intermediate phase when the spiral is distorted too much.

The spiral is distorted more and more as $H$ increases. Finally, when $H>H_{c}^{(1)}=1.65 \mathrm{~T}$, just distorting a $2 \mathrm{D}$ spiral is

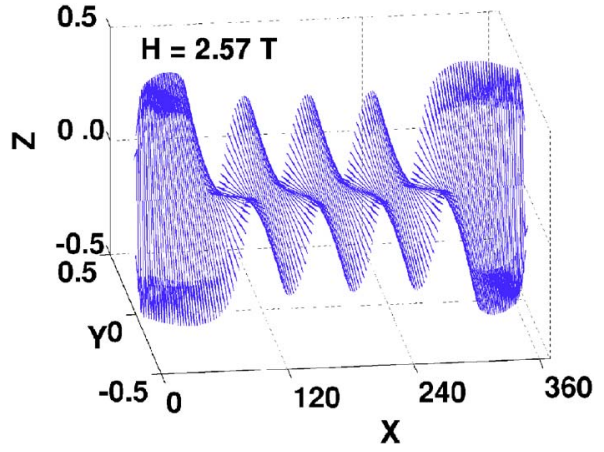

FIG. 5. (Color online) 3D view of spin configuration obtained by SD simulations at $H=2.57 \mathrm{~T}$. The calculations were performed on a $370 \times 8$ lattice, and here we only depict the spin configuration along one chain.

not enough to relax the effect of the $H$ field. The system then enters the intermediate phase. Figure 5 shows a three-dimensional (3D) view of the spin configurations in the case of $H=2.57 \mathrm{~T}$, which is a typical example inside the intermediate phase. Obviously, the ground state is now a mixing of a spiral, located in the middle, and two spin-flop states located in two boundaries. In addition, the spiral is no longer a $2 \mathrm{D}$ one, but becomes a 3D one signaled by the appearance of $S^{y}$ component.

To gain a better understanding, we show the projected spin configurations for three spin components in Figs. 6(a)-6(c), respectively. The pattern of $S^{x}$ [Fig. 6(a)] manifests clearly that the ground state is a soliton, which links two spin-flop states by a 3D spiral state. The fact that the spiral is 3.5 periods long suggests that the two spin-flop states must be out of phase. This is a very typical feature of a standard soliton solution. ${ }^{17}$

It is interesting to note that the $S^{z}$ components are greatly suppressed in this state, and those missing $S^{z}$ components are transferred to the $S^{y}$ components. In fact, the $H$ field has a very subtle effect on the spin polarization along the $z$ direc-

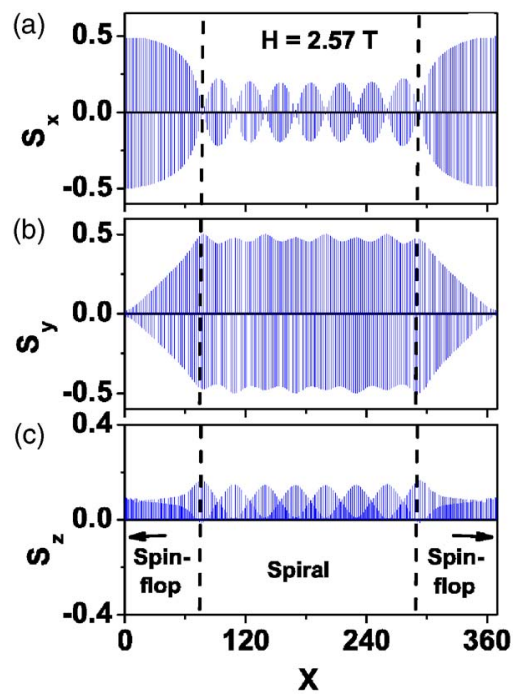

FIG. 6. (Color online) Projected spin configurations for three spin components at $H=2.57 \mathrm{~T}$. 


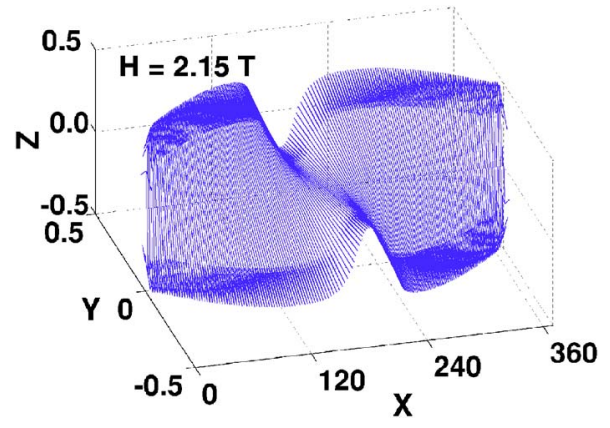

FIG. 7. (Color online) 3D view of the spin configuration obtained by SD simulations with $H=2.75 \mathrm{~T}$. The calculations were performed on a $370 \times 8$ lattice, and here we only depict the spin configuration along one chain.

tion. A naïve understanding from the Zeeman interaction (5) suggests that a $H$ field should favor a large $S^{z}$ value. However, things are quite different and complicated in such a frustrated spin system. In the absence of a $H$ field, the ground state is a spiral so that the spin components along the $x$ and $z$ directions are equal (see Fig. 3). When $H$ is large enough to destroy the spiral state, but not enough to compete with the antiferromagnetic exchange $J$, the competition between $H$ and $J$ results in a spin-flop state in which the spins are almost parallel to the $x$ axis leaving some components along the $z$ direction (see the spin-flop regime in Fig. 3). Thus, quite contrary to an intuitive expectation, the effect of a $H$ field is to suppress $S^{z}$ value in this situation. From Fig. 3, we have seen precisely that the $S^{z}$ component decreases through distorting a usual spiral state as $H$ increases in the spiral regime. However, when $H$ is so large that just distorting a spiral is not enough to reflect this tendency, the appearance of $S^{y}$ component is another choice to decrease the $S^{z}$ component. This is the inherent physics accounting for the appearance of a 3D spiral in the intermediate phase.

As $H$ increases, the total length of the 3D spiral regime in the soliton shrinks so that the spiral period decreases slightly ( $\zeta$ increases slightly accordingly). However, when $H$ increases to some specific points, the 3D spiral loses one period abruptly, resulting in a sudden increase of the spiral period (and thus a sudden decrease in $\zeta$ ). For the specific example shown in Figs. 5 and 6, the spiral loses a period abruptly and transits to a 2.5 -period state at $H=2.62 \mathrm{~T}$. This process continues as $H$ increases, until $H=H_{c}^{(2)}$ when the system departs from the intermediate phase and enters the spin-flop phase. Figure 7 shows a 3D view of the spin configuration at $H=2.75 \mathrm{~T}$ (e.g., right before the phase transition). From the projected spin configuration for $S^{x}$ shown in Fig. 8(a), we find that the spiral regime has only 0.5 period. The half period is the precise requirement to ensure that the two spin-flop states are out of phase. ${ }^{17}$ When $H$ increases further, such a soliton configuration cannot be sustained, and the system then transits to a purely commensurate spin-flop state.

We emphasize that this step-like $\zeta-H$ relation is a typical feature of a finite-sized system. However, we expect the $\zeta$ $H$ relation will become smooth in a realistic experiment, since the studied system is much larger and thermal fluctua-

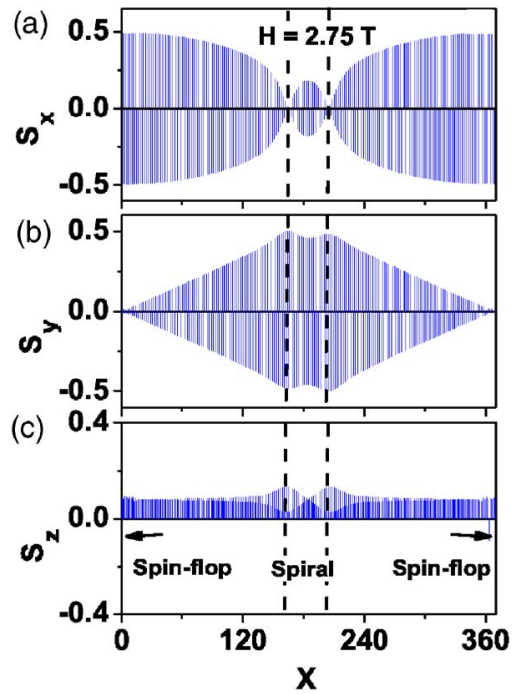

FIG. 8. (Color online) Projected spin configurations for three spin components at $H=2.75 \mathrm{~T}$.

tions are inevitable to smear all step-like features.

Although the results shown in Figs. 5-8 were all obtained with a relatively short sample (length $=370 a$ ), we note that the qualitative conclusions drawn here (i.e., soliton, 3D spiral, the intermediate phase, etc.) are independent of the sample length. For example, we show, in Fig. 9, the projected spin configuration at $H=2.57 \mathrm{~T}$, calculated with a longer sample (length $=1480 a$ ). Obviously, the ground spin configuration is still a soliton with a 3D spiral located in between two spin-flop states. This conclusion remains valid for even longer samples (up to length $=3000 a$, according to our computations). To make the whole story complete, we have calculated the size dependences of the two critical fields, $H_{c}^{(1)}$ and $H_{c}^{(2)}$, and have shown the results in Fig. 10. The fact that $H_{c}^{(1)}$ and $H_{c}^{(2)}$ are nearly independent of the sample size indicates that the intermediate phase is an intrinsic property of the system, rather than a finite-size artifact.

\section{SUMMARIES AND CONCLUSIONS}

With the help of SD simulations, we have presented here a systematic study of the field-induced incommensurate-tocommensurate phase transition for the spiral antiferromagnet

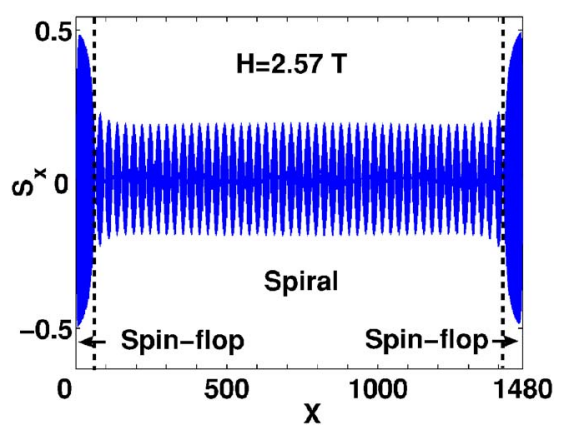

FIG. 9. (Color online) Projected spin configuration along the $x$ direction at $H=2.57 \mathrm{~T}$ calculated on a $1480 \times 8$ lattice. 


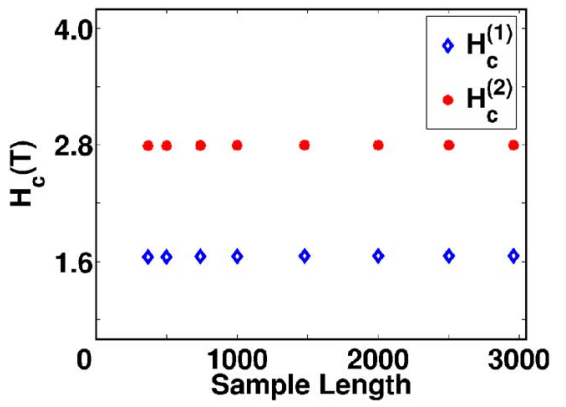

FIG. 10. (Color online) Critical fields $\left(H_{c}^{(1)}, H_{c}^{(2)}\right)$ as the functions of the sample length adopted in the SD simulations.

$\mathrm{Ba}_{2} \mathrm{CuGe}_{2} \mathrm{O}_{7}$. Our results confirmed the 2D spiral-like spin configuration in the low field regime and the spin-flop states in the high field regime, which have been understood by previous experiments and continuum-model theories. What is unclear from previous studies is the nature of the intermediate phase, which has been revealed by our simulations as a soliton-like solution linking two spin-flop states by a 3D spin spiral. The appearance of $S^{y}$ is identified as a crucial character of the intermediate phase. Moreover, we have illustrated the complete process of how the spin configuration develops from the $2 \mathrm{D}$ spiral through the intermediate phase to the spin-flop state. Some conjectures proposed previously for the intermediate phases, such as the soliton state and a nonflat (i.e., 3D) spin spiral, have been verified by our brute-force simulations.

\section{ACKNOWLEDGMENTS}

This work was supported in part by RGC CERG Grant No. HKUST 6035/03P. L.Z. received support from the National Basic Research Program of China (No. 2004CB719800), National Natural Science Foundation of China (No. 10504003), the Program for Changjiang Scholars and Innovative Research Team in University, and the Shanghai Pujiang Program.
*Author to whom correspondence should be addressed. Email address: phzhou@fudan.edu.cn

${ }^{1}$ I. E. Dzyaloshinskii, Sov. Phys. JETP 20, 665 (1965).

${ }^{2}$ T. Moriya, Phys. Rev. 120, 91 (1960).

${ }^{3}$ I. E. Dzyaloshinskii, Sov. Phys. JETP 5, 1259 (1957).

${ }^{4}$ A. Zheludev, G. Shirane, Y. Sasago, N. Koide, and K. Uchinokura, Phys. Rev. B 54, 15163 (1996).

${ }^{5}$ A. Zheludev, S. Maslov, G. Shirane, Y. Sasago, N. Koide, K. Uchinokura, D. A. Tennant, and S. E. Nagler, Phys. Rev. B 56, 14006 (1997).

${ }^{6}$ A. Zheludev, S. Maslov, G. Shirane, Y. Sasago, N. Koide, and K. Uchinokura, Phys. Rev. Lett. 78, 4857 (1997).

${ }^{7}$ A. Zheludev, S. Maslov, G. Shirane, Y. Sasago, N. Koide, and K. Uchinokura, Phys. Rev. B 57, 2968 (1998).

${ }^{8}$ A. Zheludev, S. Maslov, G. Shirane, I. Tsukada, T. Masuda, K. Uchinokura, I. Zaliznyak, R. Erwin, and L. P. Regnault, Phys. Rev. B 59, 11432 (1999).

${ }^{9}$ For a review on the incommensurate to commensurate phase tran- sition, see Per Bak, Rep. Prog. Phys. 45, 587 (1982).

${ }^{10}$ J. Chovan, N. Papanicolaou, and S. Komineas, Phys. Rev. B 65, 064433 (2002).

${ }^{11}$ W. F. Brown, Magnetostatic Principles in Ferromagnetism (Springer, New York, 1966).

${ }^{12}$ W. F. Brown, Jr., Micromagnetics (Wiley Interscience, New York, 1963).

${ }^{13}$ L. Landau and E. Lifshitz, Phys. Z. Sowjetunion 8, 153 (1935).

${ }^{14}$ E. Weinan and X. P. Wang, SIAM (Soc. Ind. Appl. Math.) J. Numer. Anal. 38, 1647 (2001).

${ }^{15}$ X. P. Wang, Carlos J. Garcia-Cervera, and E. Weinan, J. Comput. Phys. 171, 357 (2001).

${ }^{16}$ The variation of the incommensuration as a function of field was touched upon first by T. Nagamiya, K. Nagata, and Y. Kitano, Prog. Theor. Phys. 27, 1253 (1962).

${ }^{17}$ P. G. Drazin and R. S. Johnson, Solitons: An Introduction (Cambridge University Press, New York, 1989). 